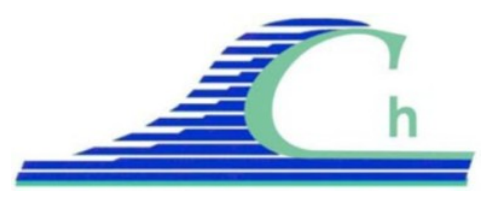

XII ${ }^{\text {èmes }}$ Journées Nationales Génie Côtier - Génie Civil

Cherbourg, 12-14 juin 2012

DOI:10.5150/jngcgc.2012.047-S @ Editions Paralia CFL

disponible en ligne - http://www.paralia.fr - available online

\title{
Interactions haut de plage - barres pré-littorales
}

\author{
Nadia SENECHAL ${ }^{1}$, Christina PERON ${ }^{1}$ \\ 1. Université Bordeaux, UMR EPOC 5805, avenue des facultés, 33405 Talence, France. \\ n.senechal@epoc.u-bordeaux1.fr ; Christina.Peron@univ-littoral.fr
}

\section{Résumé :}

Cette étude présente l'analyse de l'évolution d’un proxy du trait de côte défini comme la position de l'iso-contour associé aux marées hautes de mortes-eaux sur une période de 3 ans à partir de données vidéo quotidiennes. La réponse de ce proxy est présentée conjointement avec la dynamique de la barre interne prélittorale. Le site d'étude est situé sur la côte aquitaine, à Biscarrosse, plage ouverte linéaire soumise aux houles océaniques. On observe une dynamique saisonnière aussi bien au niveau de ce proxy que de la barre interne avec des hivers caractérisés par un recul du trait de côte (environ $40 \mathrm{~m}$ ) et une migration vers le large de la barre (environ $100 \mathrm{~m}$ ) et des périodes estivales caractérisées par une reconstruction de la plage. La réponse à court terme aux évènements très énergétiques (Hs>4 m) est complexe et variée. On observe aussi bien des évènements érosifs brutaux que des périodes de ré-engraissement rapides. Au niveau du trait de côte, cette complexité s'explique notamment par la nature des interactions avec la barre interne, celle-ci pouvant dissiper ou non l'énergie de la houle incidente, ou encore agir ou non comme un stock sédimentaire rapidement mobilisable.

Mots-clés :

Trait de côte - Tempêt - Post-tempête - Erosion - Barres prélittorales - Imagerie vidéo

\section{Abstract:}

Daily video surveys of a $1.0 \mathrm{~km}$ stretch of an open sandy beach at Biscarrosse are presented to analysis seasonal and short-term dynamic of shoreline position, defined as the mean high tide position associated to neap tide conditions, together with inner bar dynamic. While the seasonal pattern is observed, short-term dynamic associated to storm and post-storm events is more complex. This complexity can be partly explained by the kind of interactions observed between the beach face and the innner bar that can either dissipate the wave energy or not, or act as a sediment supply for rapid beach reconstruction.

\section{Keywords:}

Shoreline - Video survey - Storm - Post-storm - Erosion - Nearshore bar

\section{Introduction}

Le littoral ne peut plus être considéré de nos jours comme une zone tampon. La forte pression socio-démographique et économique qui s’y exerce, le rend vulnérable en particulier à toute fluctuation de la position du trait de côte en relation avec les 
changements climatiques. Prévoir l'évolution du trait de côte à court, moyen et longterme est donc devenu un enjeu important pour les gestionnaires et a ainsi fait dernièrement l'objet d'un atelier de travail dans le cadre des 1ères Assises Nationales des Risques Naturels (Bordeaux, 19 \& 20 janvier 2012).

D'un point de vue scientifique, les processus qui contrôlent l'évolution du trait de côte sont complexes et dépendent des échelles de temps et d'espace considérées (STIVE et al., 2002). Dans le cas des plages sableuses présentant des systèmes de barres prélittorales, l'importance des interactions et couplages entre les différentes entités morphologiques a dernièrement encore été soulignée dans la littérature (CASTELLE et al., 2010 ; PRICE et al., 2011). Les techniques de rechargement de l'avant-côte pour limiter les phénomènes de recul de trait de côte sont d'ailleurs de plus en plus utilisées (BARNARD et al., 2009). De récentes études soulignent la nécessité de prendre en considération les interactions entre le haut de plage et les systèmes de barres prélittorales, incluant celles situées en bas de plage (e.g. SONU, 1973 ; THORNTON et al., 2007 ; SENECHAL et al., 2009 ). Toutefois, en comparaison au nombre de travaux s'intéressant à l'influence des rechargements de sable de l'avant côte sur l'évolution du trait de côte (par exemple le projet NOURTEC), peu d'études ont porté sur la réponse du haut de plage en lien avec la configuration naturelle des barres situées en bas de plage.

Dans cette étude, nous analysons la réponse journalière du haut de plage d'une plage sableuse à barres conjointement avec la dynamique des barres pré-littorales. L'étude repose sur des données vidéo horaires acquises sur une période de 3 années consécutives. Un évènement majeur d'alimentation du haut de plage par transfert sédimentaire du système de barres vers la côte est présenté.

\section{Matériel et Méthode}

\subsection{Le site d'étude}

La plage de Biscarrosse se situe sur le littoral aquitain à environ $10 \mathrm{kms}$ au sud de l'embouchure du Bassin d'Arcachon (figure 1). Elle est constituée de sable moyen et le pied de dune de la plage centrale a été stabilisé par des géotextiles suite à la tempête Klaus en 1999. Elle se caractérise par un système de double barres (ALMAR et al., 2009). La barre subtidale est située à environ 400 mètres de la ligne de rivage à marée haute et présente généralement une forme en croissants caractéristique de la ligne de barres subtidales observées le long de la côte aquitaine (LAFON et al., 2004) qui, sous l'effet d'une forte tempête peut se linéariser (ALMAR et al., 2010). La barre intertidale se caractérise quant à elle principalement par une configuration de type TBR (Transverse Bar and Rip) même si tous les états intermédiaires sont observés (PERON \& SENECHAL, 2011). Malgré des apports sédimentaires réguliers provenant des Passes d'Arcachon, la plage de Biscarrosse reste en recul depuis quelques années. 


\section{XII ${ }^{\text {èmes }}$ Journées Nationales Génie Côtier - Génie Civil \\ Cherbourg, 12-14 juin 2012}

D'un point de vue hydrodynamique, la plage de Biscarrosse est soumise à un régime méso-macrotidal avec un marnage moyen lors des vives-eaux de $3.7 \mathrm{~m}$. Elle est soumise aux houles de l'Atlantique Nord dont la hauteur moyenne annuelle pendant l'étude était de l'ordre de 1.66 m (PERON \& SENECHAL, 2011).

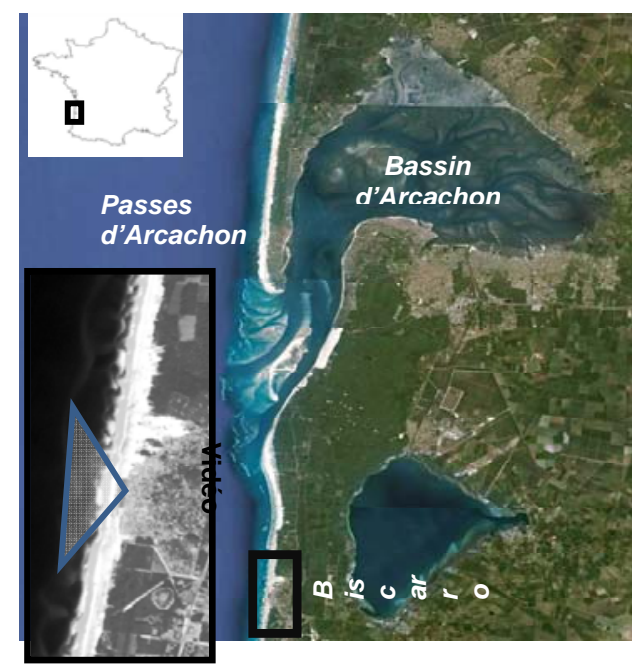

Figure 1. Site d'étude, Biscarrosse sur la côte Aquitaine.

\subsection{Données et méthode}

Les données hydrodynamiques ont été obtenues à partir des modèles tidaux du SHOM pour la marée ; et du modèle Wave Watch 3 et de la bouée Cap Ferret (CETMEF CANDHIS) pour les caractéristiques des vagues au large (Hs, Tp et Dp).

Concernant les données morphologiques, elles ont été obtenues à partir des données vidéo horaires. Afin de suivre la dynamique du haut de plage, nous nous sommes appuyés sur une définition d'un proxy du trait de côte généralement utilisée dans ce type d'étude (MOORE et al., 2006). Ainsi la position de l'iso-contour correspondant à l'altitude moyenne des hautes mers de mortes-eaux a été extraite des images vidéo moyennées sur 10 minutes redressées et géo-référencées. Cette extraction s’est faite en positionnant la ligne d'eau moyenne lorsque l'altitude du plan est à la côte voulue (figure 2). Les journées sélectionnés l'ont été de telle manière à négliger les erreurs d'estimation de l'altitude du plan d'eau liées au forçage météorologique (baromètre inverse et vent) par rapport au set-up des vagues estimé à partir des formules proposées par STOCKDON et al. (2006). L'erreur sur la position de la ligne d'eau peut être estimée à $+/-3 \mathrm{~m}$ en moyenne pour les cas où la hauteur significative des vagues est inférieure à $2.0 \mathrm{~m}$ et $+/-6 \mathrm{~m}$ en moyenne lors des tempêtes. L'erreur maximale lors des tempêtes se traduit par une surestimation du recul de la ligne d'eau qui peut atteindre $10 \mathrm{~m}$. A partir de cette extraction, nous avons ensuite déduit une position moyenne et évalué la tridimensionnalité de cette ligne d'eau en calculant l'écart type associé. Cette estimation s'est faite sur un linéaire côtier d'environ $1 \mathrm{~km}$. 


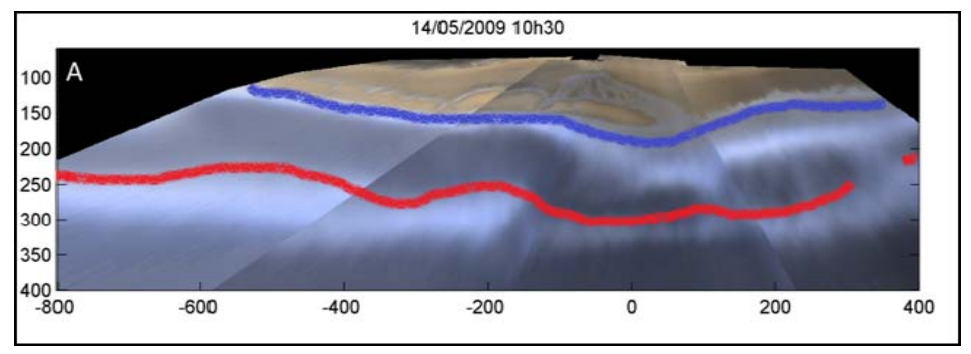

Figure 2. Extraction de la ligne d'eau (bleu) et de la position de la barre (rouge).

La position des barres pré-littorales a été obtenue en localisant la zone de déferlement (en blanc sur la figure 2) à partir d'images vidéo obtenues pour la même altitude et des conditions de vagues similaires. Les journées pour lesquelles, il n’y avait pas de déferlement ou pour lesquelles la qualité d'image n'était pas correcte, n’ont pas été retenues. De la même façon, nous en avons déduit une position moyenne et un facteur de tridimensionnalité à partir de l'écart type associé. L'erreur sur la barre interne pouvant atteindre $+/-10 \mathrm{~m}$.

\section{Résultats et discussion}

La figure 3 représente la hauteur des vagues au large (par environ $50 \mathrm{~m}$ de fond), la position de la barre externe subtidale, de la barre interne et de la ligne d'eau. La position est celle mesurée par rapport à l'origine du repère fixé arbitrairement comme étant la position du système vidéo situé au sommet de la dune prélittorale. On observe clairement le caractère saisonnier du forçage hydrodynamique dans cette zone avec des périodes hivernales plus énergétiques associées à des évènements de tempête où la hauteur significative des vagues peut dépasser plusieurs fois $5 \mathrm{~m}$ sur des durées de l’ordre de la journée et au contraire des saisons estivales beaucoup moins énergétiques.

\subsection{Dynamique de la barre interne et de la ligne d'eau sur les 3 années}

La figure 3 met également en évidence la dynamique de la barre interne qui peut migrer rapidement vers le large lors d'évènements de tempête (dizaine de mètres / jour), puis migrer vers la côte plus lentement (mètre/jour); ces ordres de grandeur sont cohérents avec les observations faites dans des environnements similaires (VAN ENCKEVORT et al., 2004 ; ALMAR et al., 2009).

Sur l'ensemble de la période, la barre interne se situe généralement entre les positions $300 \mathrm{~m}$ et $150 \mathrm{~m}$. Le haut de plage présente également une dynamique notable avec des périodes d'engraissement associées à une migration vers la large de la position de la ligne d'eau, généralement observées pendant les périodes estivales ou faiblement énergétiques et des périodes d'amaigrissement de la plage généralement observées pendant la période hivernale. Ces résultats sont en accord avec de précédentes études réalisées sur le site du Truc Vert à partir de levés GPS (SENECHAL et al., 2009). 


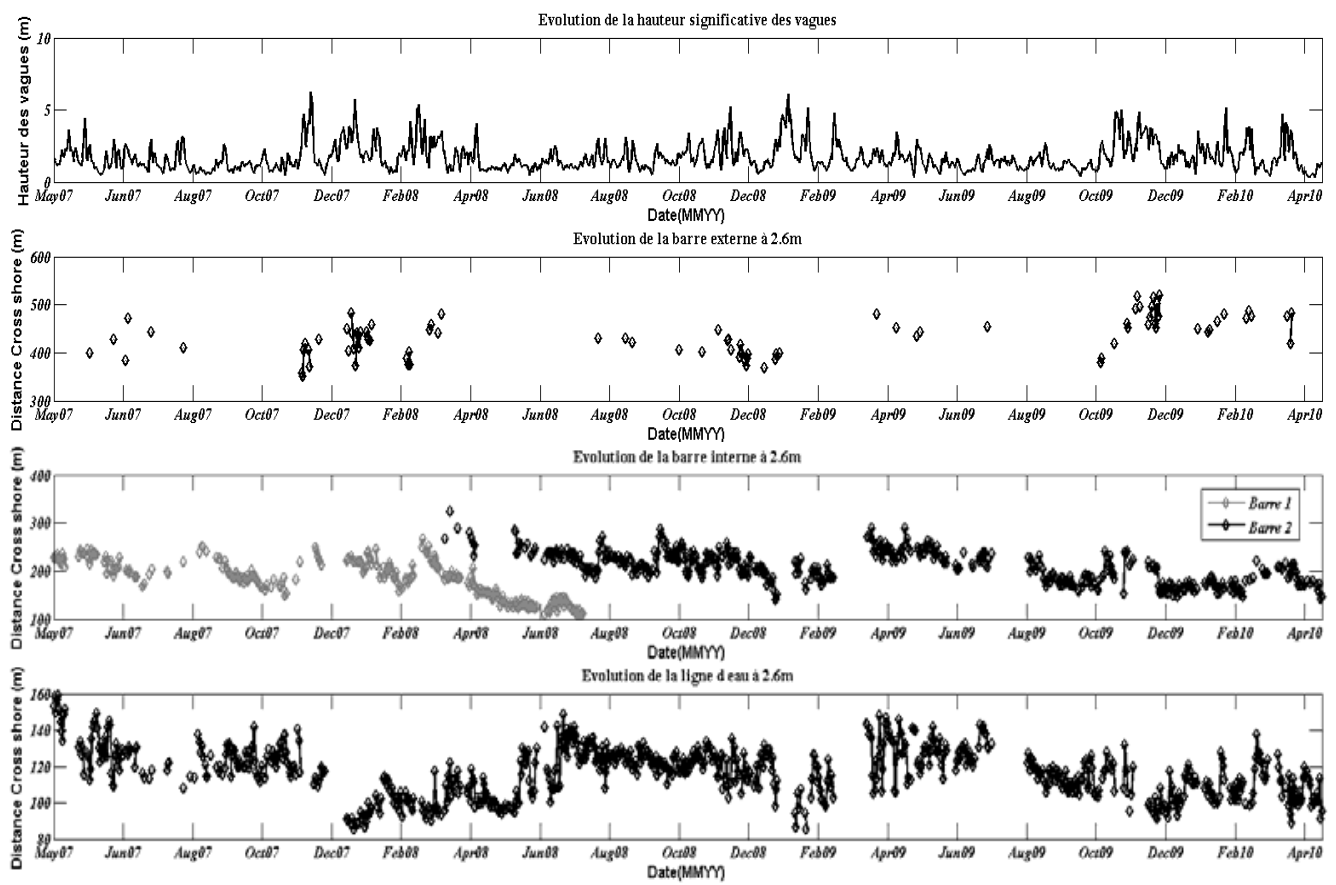

Figure 3 : évolution de la hauteur significative des vagues, de la position moyenne de la barre externe, des barres internes (généralement une seule est observée) et de la ligne d'eau. La position est donnée par rapport au système vidéo déployé sur le sommet de la dune prélittorale.

\subsection{Episodes novembre 2007 - mai 2008}

Cette série d'épisodes est intéressante car elle montre que la réponse de la barre et du haut de plage, même si elle répond à moyen terme à des cycles saisonniers soulignés précédemment, a une réponse aux évènements de tempêtes et post-tempêtes relativement complexes, variées et rapides.

Ici ne serons représentés que les hauteurs significatives des vagues, les périodes et direction étant relativement similaires pour ces différents évènements. En novembre 2007, la première tempête hivernale faisant suite à une longue période de vagues faiblement énergétiques est associée à des $\mathrm{Hs}>4 \mathrm{~m}$ observées pendant près de 48 heures (figures 3 et 4). L'impact de cette tempête se traduit par une migration rapide de la barre interne mais un haut de plage relativement préservé (figure 4). La réponse rapide de la barre interne peut être liée au fait que sa configuration était éloignée du profil d'équilibre pour de telles conditions (GRASSO et al., 2009) et de ce fait la tempête a généré un réajustement rapide de cette morphologie. La barre interne semble avoir joué ici un rôle protecteur et préserve le haut de plage d'une érosion massive en dissipant l'énergie de la houle avant son arrivée sur le haut de plage.

En revanche l'épisode suivant (décembre 2007- janvier 2008), bien que moins énergétique dans un premier temps se traduit dès le début par une érosion importante du haut de plage puis par une relative stabilité lorsque le pic énergétique intervient (voir 
légère accrétion, on rappelle que la méthode peut montrer un recul 'artificiel' d'environ $10 \mathrm{~m}$ compte tenu des conditions) alors que le système de barre interne est plutôt stable pendant toute la période. Une analyse plus détaillée des images permet de mettre en évidence que la barre interne n'est pas toujours active. En effet, lorsque les premières houles, modérément énergétiques, arrivent, la barre interne est à priori trop loin pour dissiper celles-ci (de mi-marée à marée-haute) et de ce fait celle-ci déferlent directement sur le haut de plage. En revanche lorsque les vagues forcissent, la barre interne redevient active, préservant le haut de plage.
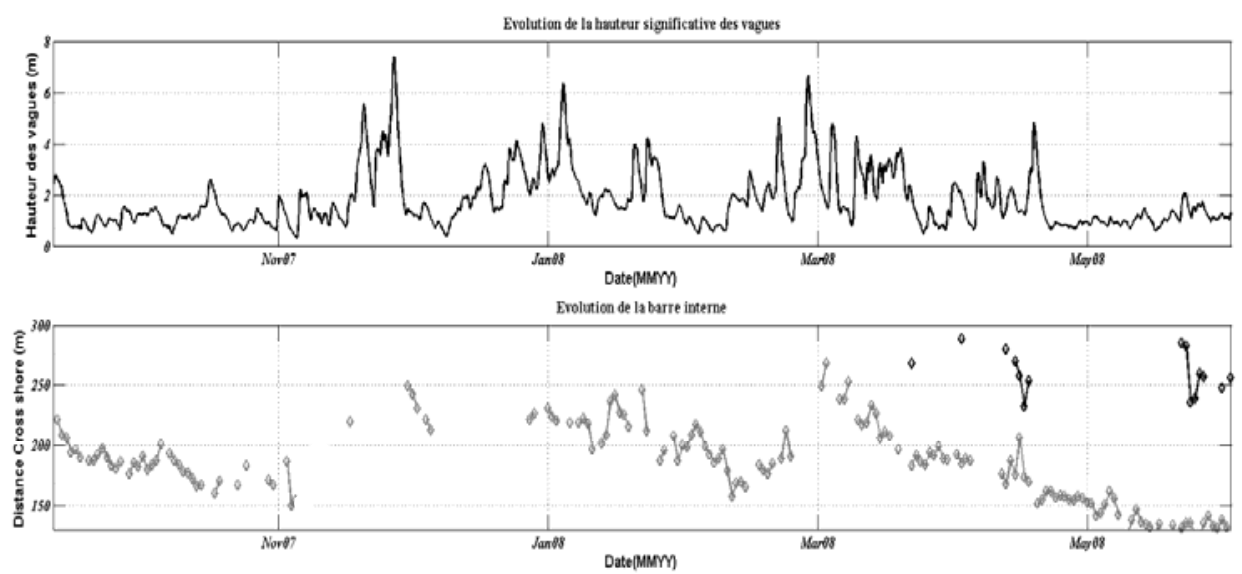

Evolution de la ligne d ear

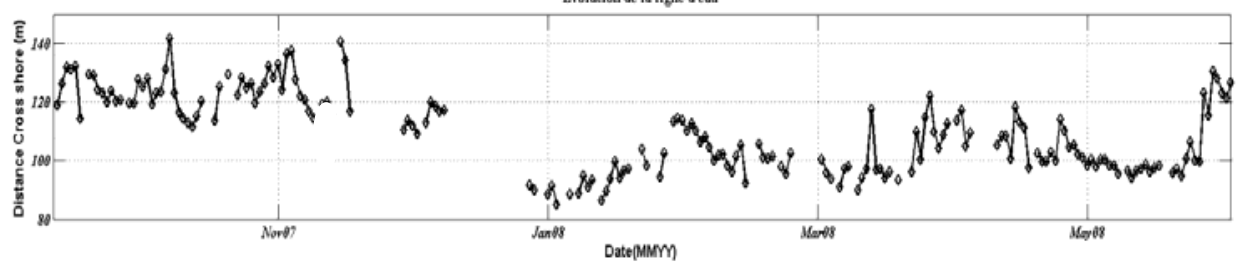

Figure 4. Zoom sur la période novembre 2007- mai 2008. Le recul de la ligne d'eau vers la côte de même que celui de la barre interne vers le large peut être surestimé lorsque $\mathrm{Hs}>2 \mathrm{~m}$ (au maximum de $10 \mathrm{~m}$ ).

On note ensuite un évènement marquant avec l'apparition d'une seconde barre interne en Mars 2008 qui se forme au large de la première barre lors de cet épisode énergétique. Ces observations corroborent le modèle conceptuel proposé par CASTELLE et al. (2007) et peuvent être mises en parallèle des observations détaillées par ALMAR et al. (2009 ; 2010). L'apparition de cette seconde barre intertidale résulterait du détachement des cornes de la barre subtidale et de leur migration vers la côte même si la séquence ne semble pas être tout à fait la même. Dans le cas détaillé par ALMAR et al. (2010), la corne s'est rapidement accolée à la barre interne alors qu'ici cela ne semble pas être le cas. Au contraire le double système de barre semble perdurer jusqu'à l'accolement de la barre interne au haut de plage, fonctionnant comme une source sédimentaire pour le 


\section{XII ${ }^{\text {èmes }}$ Journées Nationales Génie Côtier - Génie Civil \\ Cherbourg, 12-14 juin 2012}

haut de plage. Cela se traduit par un engraissement rapide de celui-ci et une avancée rapide de la ligne d'eau en mai 2008.

Au final, malgré un retour rapide de la ligne d'eau à sa position d'avant-tempête, corroborant les observations de ANDERSEN et al. (2010), une analyse plus longue reste nécessaire pour évaluer l'impact de cette séquence énergétique à plus long terme. En effet, on observe qu'à l'issue de la période d'étude ( 3 ans), la ligne d'eau est globalement en retrait.

\section{Conclusions}

L'étude de données vidéo quotidiennes associée à des données de houle au large a permis d'analyser la réponse naturelle d'un système haut de plage/barre prélittorale sur une durée de 3 ans entre 2007 et 2010. On observe sur cette période qu'il y a des périodes d'engraissement et d'érosion à l'échelle saisonnière mais que la plage réagit également rapidement aux épisodes de tempêtes, aussi bien en terme érosif qu'en terme de reconstruction. Une analyse des données sur une période plus longue reste néanmoins nécessaire pour mieux appréhender l'impact de ces évènements sur la dynamique long terme. L'analyse de ce jeu de données permet également de souligner de nouveau l'importance d'étudier les interactions et couplage entre le haut de plage et la barre interne. Cette dernière peut agir comme un brise- lames, dissipant l'énergie de la houle avant qu'elle n'arrive à la côte ou comme un stock sédimentaire rapidement mobilisable pour la reconstruction du haut de plage. Les résultats présentés ici ne montrent que l'évolution globale et moyennée le log du linéaire côtier. Une analyse sur les ondulations le long de la côte est en cours.

Remerciements : Le système vidéo Biscarrosse a été financé par la région Aquitaine et l'OASU. Nous remercions H. Wennekes, J.-M. Escalier et V. Marieu pour leur soutien logistique et leur aide dans le développement des scripts d'analyse.

\section{Références bibliographiques}

ALMAR R., CASTELLE B., RUESSINK B.G., SENECHAL N., BONNETON P., MARIEU V. (2009). High-frequency video observation of two nearby double-barred beaches under high-energy wave forcing. Journal of Coastal Research, SI56(2), pp 1706-1710.

ALMAR R., CASTELLE B., RUESSINK B.G., SEECHAL N., BONNETON P., MARIEU V. (2010). Two- and three-dimensional double-sandbar system behaviour under intense wave forcing and a meso-macro tidal range. Cont. Shelf Res., Vol. 30, pp 781-792. doi:10.1016/j.csr.2010.02.001

ANDERSEN T.R., FRAZER L.N., FLETCHER C.H. (2010). Transient and persistent shoreline change from a storm. Geophys. Res. Letters, 37, L08401. doi:10.1029/2009GL042252 
BARNARD P., ERIKSON L. H, HANSEN J. (2009). Monitoring and modeling shorenline response due to shoreface noursihment on a high-energy coast. J. Coastal Res., SI56, pp 29-33.

CASTELLE B., BONNETON P., DUPUIS H., SENECHAL N. (2007). Double bar beach dynamics on the high-energy meso-macrotidal French Aquitanian coast: a review, Marine Geology, Vol. 245, pp 141-159. doi:10.1016/j.margeo.2007.06.001

CASTELLE B., RUESSINK B.G., BONETON P., MARIEU V., BRUEAU N., PRICE T.D. (2010). Coupling mechanisms in double sandbar systems, Part 1 : Patterns and physical explanation. Earth surface processes and landforms, Vol. 35, pp 476-486.

GRASSO F., MICHALLET H., BARTHELEMY E., CERTAIN R. (2009). Physical modeling of intermediate cross-shore beach morphology: transients and equilibrium states. J. Geophy. Res., Vol. 114, C09001, doi:10.1029/2009JC005308

LAFON V., De MELO D., DUPUIS H., MICHEL D., HOWA H., FROIDEFOND J.M. (2004). Morphodynamics of earshore rhythmic sandbars in a mixed-energy environment (SW France) : I. Mapping beach changes using visible satellite imagery. Estuarine Coastal ad Shelf science, Vol. 61, pp 289-299.

MOORE L.J., RUGGIERO P., LIST J.H. (2006). Comparing mean high water ad high water lie shorelies: should proxy-datum offsets be incorporated ito shorelie chage analysis? Journal of Coastal Research, Vol. 22(4), pp 894-905.

PERON C., SENECHAL N. (2011). Dynamic of a meso to macro-tidal double barred beach : inner bar response. Journal of Coastal Research, SI64, pp 120-124.

PRICE T.D., RUTTEN J., RUESSINK B.G. (2011). Coupled behavior within a double sandbar system. Journal of coastal research, SI64, pp 125-129.

SÉNÉCHAL N., GOURIOU T., CASTELLE B., PARISOT J.P., CAPO S., BUJAN S., HOWA H. (2009). Morphodynamic response of a meso- to macro-tidal intermediate beach based on a long-term data-set, Geomorphology, Vol. 107, pp 263-274. doi:10.1016/j.geomorph.2008.12.016

SONU C.J. (1973). Three-dimensional beach changes. J. of Geology, Vol. 81, pp 42-64. doi:10.1086/627806

STIVE M.J.F., AARNINKHOF S.G.J. HAMM L., HANSEN H., LARSON M., WIJNBERG K.M., ICHOLLS R.J., COPOBIANCO M. (2002). Variability of shore and shoreline evolution. Coast Engineering, Vol. 47(2), pp 211-235. doi:10.1016/S03783839(02)00126-6

STOCKDON H.F., HOLMAN R.A., HOWD P.A., SALLENGER JR A.H. (2006). Empirical parameterization of setup, swash and runup. Coastal Engineering, Vol. 53, pp 573-588. doi:10.1016/j.coastaleng.2005.12.005

THORNTON E.B., MACMAHAN J., SALLENGER JR A.H. (2007). Rip currents, mega-cusps, and eroding dunes. Marine Geology, Vol. 240, pp 151-167. doi:10.1016/j.margeo.2007.02.018. 